\title{
La Inteligencia emocional en la Educación
}

\author{
Pablo Fernández-Berrocal, Desiree Ruiz Aranda
}

Facultad de Psicología, Universidad de Málaga

\section{España}

Dr. Pablo Fernández Berrocal, Facultad de Psicología, Departamento de Psicología Básica, Universidad de Málaga, Campus de Teatinos S/N (29071). Málaga. Telf. 952-131086. Fax: 952-132631.

Email: berrocal@uma.es; desiree@uma.es

Este artículo fue realizado en parte gracias a la ayuda SCO/3386/2004 del Plan Nacional sobre Drogas (Ministerio de Sanidad y Consumo).

(C) Education \& Psychology I+D+i and Editorial EOS (Spain) 


\section{Resumen}

La Inteligencia Emocional (IE) ha surgido en los últimos 25 años como un concepto muy relevante del ajuste emocional, el bienestar personal, el éxito en la vida y las relaciones interpersonales en diferentes contextos de la vida cotidiana. Este artículo ofrece una revisión crítica de las investigaciones sobre IE en el contexto escolar y analiza su valor tanto presente como futuro para las políticas educativas en el sistema educativo español.

En primer lugar, los autores examinan el debate surgido en diferentes países (como por ejemplo, Reino Unido, estados Unidos y España) sobre las políticas educativas para facilitarles a los niños el mejor comienzo en sus vidas y el desarrollo de sus habilidades de IE. En segundo lugar, presentan los modelos teóricos de IE y decriben con detalle el modelo de Mayer y Salovey (1997). En tercer lugar, los autores resumn la investigación referente a la relevancia de la IE a los indicadores del éxito personal y escolar como las relaciones interpersonales, el éxito académico, y el ajuste personal y social. Finalmente, concluyen con algunas recomendaciones sobre el desarrollo de la IE en la escuela y algunas implicaciones sobre las futuras políticas educativas en España.

Palabras Clave: inteligencia emocional, ajuste personal, éxito académico, políticas educativas. 


\begin{abstract}
Emotional intelligence (EI) has emerged in the past twenty five years as one of the crucial components of emotional adjustment, personal well-being, life success, and interpersonal relationship in different contexts of everyday life. This article provides a critical review of the research field of EI in the school context and analyzes its present and future value for educational policies in the Spanish educational system.

First, the authors examine the debate on educational policies to provide children the best posible start in life in different countries (e.g., United Kingdom, United States and Spain), and the development of EI abilities. Second, they present theoretical models of EI and describe in detail the Mayer and Salovey model (1997). Third, the authors summarize research concerning the relevance of EI to personal and school success indicators as interpersonal relationship, academic achievement, and personal and social adjustment. Finally, they conclude with some recommendations for the development of EI in the school and several implications for future educational policies in Spain.
\end{abstract}

Keywords: Emotional intelligence, personal adjustment, school success, educational policies.

Received: 04/14/08 Initial Acceptance: 05/11/08 Final Acceptance: 05/23/08 


\section{Introducción}

"He comprobado con frecuencia, y con profunda pena, que existe una prevención frecuente contra la educabilidad de la inteligencia. El proverbio de familia que dice que «cuando uno es bestia lo es para mucho tiempo» parece tomarse al pie de la letra por maestros sin crítica; éstos se desinteresan de los alumnos a quienes falta inteligencia; no tienen para ellos ni simpatía ni siquiera respeto, porque su intemperancia de lenguaje les hace decir delante de tales niños cosas como ésta: "Es un muchacho que nunca servirá para nada... está mal dotado, no tiene inteligencia». Yo he oido muchas veces estas palabras imprudentes. Cotidianamente se repiten en las escuelas primarias y también en las secundarias. Recuerdo que en mi examen del bachillerato en letras, el examinador Martha, indignado por una de mis respuestas (yo había dado a un filósofo griego, por confusión de palabras, un nombre arrancado a uno de los personajes de los Caracteres, de La Bruyère), me declaró que no tendría nunca el espíritu filosófico. ¡Nunca! ¿Qué palabra más atrevida! Algunos filósofos recientes parecen haber dado su apoyo moral a estos veredictos deplorables, afirmando que la inteligencia de un individuo es una cantidad fija, una cantidad que no puede aumentar. Debemos protestar y reobrar contra este pesimismo brutal; vamos a demostrar que no se funda en nada." (Alfred Binet, 1909).

Un siglo después de estos pensamientos de Alfred Binet (1909) seguimos preocupados por cómo lograr que los alumnos mejoren tanto sus capacidades intelectuales como su rendimiento académico. Por ello, es curioso que Binet sea más famoso en nuestras aulas universitarias por inventar el concepto de Cociente Intelectual (CI) que por sus intentos casi desesperados por diseñar programas educativos para, diríamos hoy, integrar a los niños con dificultades intelectuales y del aprendizaje.

No obstante, si analizamos la cita de Binet desde un punto de vista más psicológico encontramos que la anécdota que narra es un recuerdo emocional personal muy negativo. En concreto, el comportamiento de su profesora Martha cuando él estaba realizando su examen de bachillerato. La atrevida, dice educadamente Binet (imprudente, preferimos decir nosotros), palabra “¡Nunca!” retumba aún en sus oídos cuando escribe Las ideas modernas acerca de los niños al final de su vida (muere en 1911). Por fortuna, el pequeño Alfred no atendió a las imprudentes y desalentadoras palabras de su profesora y se convirtió años después en catedrático en La Sorbonne y en un pensador original y muy influyente. En la actualidad, Alfred 
Binet es considerado uno de los padres de la psicología moderna con más de 115,000 entradas en el buscador Google.

Por desgracia, no todos los niños que reciben en la escuela o en su familia estas "atrevidas" afirmaciones sobre, por ejemplo, sus capacidades intelectuales o físicas son capaces de sobreponerse emocionalmente a sus efectos. Las competencias emocionales y sociales necesarias para afrontar de forma adecuada las emociones negativas y destructivas que son generadas en este caso en un contexto tan competitivo como el escolar no han sido enseñadas de forma explícita en nuestra cultura. ¿Por qué? Porque nuestra sociedad y, en concreto, la escuela han priorizado hasta finales del siglo XX los aspectos intelectuales y académicos de los alumnos convencidos que los aspectos emocionales y sociales pertenecen al plano privado y, en este sentido, cada individuo es responsable de su desarrollo personal (Evans, 2002; Fernández-Berrocal y Ramos, 2002).

El siglo XXI nos ha traído una nueva forma de ver la realidad más diversa sobre el funcionamiento de las personas y estamos tomando conciencia de forma lenta, aunque progresiva, de la necesidad de que la educación de los aspectos emocionales y sociales sean atendidos y apoyados por la familia, pero también de forma explícita por la escuela y la sociedad. Las sociedades posmodernas del primer mundo en las que vivimos no quieren sólo ser ricas, opulentas y estar entre los 25 países con más PIB (Producto Interno Bruto) del mundo como ha ocurrido hasta finales del siglo XX, como señala Gilles Lipovetsky, quieren algo más. Las sociedades hipermodernas del siglo XXI, como Lipovetsky las denomina, no sólo desean el consumo material, sino que buscan una nueva jerarquía de valores y una nueva forma de relacionarse con los objetos y el tiempo, con nosotros mismos y con las personas, con el fin de encontrar la felicidad individual (Lipovetsky, 2006). Las sociedades hipermodernas buscan que sus ciudadanos estén satisfechos con sus vidas porque es una paradoja muy dolorosa para el individuo vivir en una sociedad opulenta que cubre sus necesidades físicas y materiales, pero que no le hace feliz.

Esta nueva visión la podemos encontrar de forma directa en diferentes organismos e instituciones oficiales tan relevantes como, por ejemplo, las Naciones Unidas:

"La verdadera medida del progreso de una nación es la calidad con que atiende a sus niños: su salud y protección, su seguridad material, su educación y socialización y el modo en 
que se sienten queridos, valorados e integrados en las familias y sociedades en las que han nacido" (p. 6).

Esta cita está tomada del inicio del Informe Innocenti "Pobreza Infantil en Perspectiva: Panorama del Bienestar Infantil en Países Ricos”, elaborado por Unicef sobre 21 países $h i$ permodernos. Este estudio evalúa y compara la calidad de vida de los niños en seis dimensiones: bienestar material, salud y seguridad, educación, relaciones familiares y entre iguales, conductas y riesgos, y percepción subjetiva de los propios niños. Estas dimensiones se miden con 40 indicadores independientes y significativos para la vida de los niños, según el concepto de bienestar infantil recogido en la Convención sobre los Derechos del Niño de las Naciones Unidas.

En este informe, Holanda ocupa el primer lugar de la lista en el bienestar de los menores, seguida por Suecia, Dinamarca y Finlandia. Sin embargo, países como Gran Bretaña y Estados Unidos ocupan los últimos lugares por debajo de países más pobres como Polonia o la República Checa. Este dato es muy relevante porque nos muestra que no hay una asociación lineal entre el bienestar de la infancia de un país y su PBI: la riqueza de las sociedades no garantiza la satisfacción y la felicidad de sus ciudadanos, al menos, de los más pequeños.

España ocupa una buena posición en este ranking de bienestar. Los niños y adolescentes españoles tienen una valoración subjetiva de su bienestar muy alta, en términos de percepción de su propia salud y grado de satisfacción sobre su vida. Estas variables, junto a otras analizadas, sitúan a España en el puesto número cinco de la clasificación general de bienestar infantil en 21 países.

No obstante, esta visión tan positiva de nuestro país contrasta con otros datos recientes que nos muestran que los jóvenes españoles también tienen graves problemas. En primer lugar, el número total de adolescentes entre 15 y 19 años que quedaron embarazadas de forma no deseada fue en España durante el año 2005 de 25965 (INE, 2007), de las cuales aproximadamente el 49,6 \% decidió abortar. En segundo lugar, el consumo de drogas legales en adolescentes, por ejemplo, el 44\% se ha emborrachado alguna vez durante el último mes (Ministerio Sanidad y Consumo, 2007). 
En Gran Bretaña, los pésimos resultados del informe de Unicef sobre el bienestar de sus menores han generado un gran debate sobre la incapacidad de una sociedad rica e hipermoderna para hacer felices a sus jóvenes. Una de las primeras reacciones ha sido la creación en junio de 2007 de una Secretaría de Estado para "Children, Schools and Families" (http://www.dfes.gov.uk/) con el propósito de garantizar que los niños y los jóvenes estén satisfechos y sean felices.

Una de las estrategias de este nuevo departamento se ha concretado en el apoyo activo de un movimiento a nivel nacional denominado "Social and Emotional Aspects of Learning" (SEAL; Aspectos Sociales y Emocionales del Aprendizaje, ver http://www.bandapilot.org.uk/) tanto para los niveles educativos de Primaria y Secundaria.

El movimiento SEAL está inspirado directamente de las propuestas integradoras denominas originalmente en EE.UU como "Social and Emotional Learning" (SEL; Aprendizaje Emocional y Social; ver www.CASEL.org). Los principios del SEL se proponen como un marco integrador para coordinar todos los programas específicos que se aplican en la escuela bajo el presupuesto básico de que los problemas que afectan a los jóvenes están causados por los mismos factores de riesgo emocional y social. De esta forma, la mejor manera de prevenir estos problemas específicos sería mediante el desarrollo práctico de las habilidades emocionales y sociales de los niños en un ambiente positivo y estimulante (Greenberg et al., 2003; Weissberg y O’Brien, 2004). Los programas SEL están basados en el concepto de Inteligencia Emocional (IE) desarrollado por Peter Salovey y John Mayer en 1990 (Salovey y Mayer, 1990) y difundido con gran éxito comercial por Daniel Goleman en 1995 (Goleman, 1995). No obstante, bajo la etiqueta de los programas SEL encontramos programas que entrenan habilidades básicas relacionadas directamente con la IE como la percepción emocional, la comprensión emocional o la regulación emocional y aspectos más amplios y de orden superior vinculados con la personalidad como la autoestima, la perseverancia, la asertividad o el optimismo (para una revisión ver, Zins, Weissberg, Wang, y Walberg, 2004).

En España, con la finalidad de introducir la educación de las emociones en la escuela, se ha generado todo un movimiento educativo que podemos encontrarlo con diferentes denominaciones como "Educación Emocional" o "Educación SocioEmocional". Este movimiento refleja la preocupación de los educadores por cambiar una escuela que se percibe en crisis e incapaz de afrontar los múltiples desafíos de nuestra sociedad. En este sentido, la inclusión de 
los aspectos emocionales y sociales en el currículo de sus alumnos se aprecia como una posible salida a alguno de los problemas urgentes del sistema educativo. El inconveniente principal con el que se encuentran los educadores es que no saben cómo llevar sus inquietudes a la práctica. En ese deseo admirable de cambio los docentes han abordado el problema desde la perspectiva de los trabajos divulgativos de Goleman y, ávidos por actuar, han obviado los debates académicos sobre los efectos reales y la eficacia comprobada de los programas de intervención en IE.

El propósito de este artículo es describir el modelo de IE de Mayer y Salovey y los beneficios concretos de la IE que se han encontrado en la literatura científica en el ámbito escolar con la intención final de promover la educación de la IE en la escuela.

Las implicaciones y la relevancia de la IE de los propios educadores en el sistema educativo, aspecto de suma importancia, no se tratará en este artículo ya que se trata explícitamente en otros artículos de este monográfico (ver Fernández, Palomero yTeruel, 2008; Palomera, Fernández-Berrocal y Brackett, 2008).

\section{El modelo de inteligencia emocional de Salovey y Mayer}

En la literatura especializada podemos encontrar una distinción entre aquellos modelos de IE que se focalizan en las habilidades mentales que permiten utilizar la información que nos proporcionan las emociones para mejorar el procesamiento cognitivo (denominados "modelos de habilidad") y aquellos que combinan o mezclan habilidades mentales con rasgos de personalidad tales como persistencia, entusiasmo, optimismo, etc. (denominados "modelos mixtos"). Los trabajos de investigación realizados apoyan la existencia de una IE que es considerada como una habilidad mental distinta de la inteligencia estándar, analítica (Mayer, Salovey y Caruso, 2000). La visión de los modelos mixtos es más general y algo más difusa, ya que sus modelos se centran en rasgos estables de comportamiento y en variables de personalidad (empatía, asertividad, impulsividad, etc.). En nuestro país en el ámbito educativo, ha sido el modelo teórico más extendido como fruto del éxito editorial del bestseller de Goleman.

Desde el modelo teórico de Salovey y Mayer la IE es concebida como una inteligencia genuina basada en el uso adaptativo de las emociones de manera que el individuo pueda solucionar problemas y adaptarse de forma eficaz al medio que le rodea. El modelo de habilidad 
de Mayer y Salovey considera que la IE se conceptualiza a través de cuatro habilidades básicas, que son:

"la habilidad para percibir, valorar y expresar emociones con exactitud, la habilidad para acceder y/o generar sentimientos que faciliten el pensamiento; la habilidad para comprender emociones y el conocimiento emocional y la habilidad para regular las emociones promoviendo un crecimiento emocional e intelectual” (Mayer y Salovey, 1997):

Podemos apreciar cómo entre la segunda rama (asimilación emocional) y las otras tres existe una distinción. Tanto la percepción, evaluación y expresión de las emociones (rama primera), como la comprensión emocional (rama tercera) y la regulación (rama cuarta) hacen referencia al proceso de razonar sobre las emociones, mientras que la segunda rama (asimilación emocional) incluye el uso de las emociones para facilitar el pensamiento.

\section{Beneficios de la mejora de la IE}

La literatura más reciente ha mostrado que las carencias en las habilidades de inteligencia emocional afectan a los estudiantes dentro y fuera del contexto escolar (Brackett, Rivers, Shiffman, Lerner y Salovey, 2006; Ciarrochi, Chan y Bajgar, 2001; Extremera y Fernández-Berrocal, 2003; Mestre y Fernández-Berrocal, 2007; Sánchez-Núñez, FernándezBerrocal, Montañés y Latorre, 2008; Trinidad y Johnson, 2002). Existen cuatro áreas fundamentales en las que una falta de IE provoca o facilita la aparición de problemas de conducta entre los estudiantes:

- Inteligencia emocional y las relaciones interpersonales,

- Inteligencia emocional y bienestar psicológico,

- Inteligencia emocional y rendimiento académico, y

- Inteligencia emocional y la aparición de conductas disruptivas.

\section{Inteligencia emocional y las relaciones interpersonales}

Uno de los objetivos más importantes de cualquier persona es mantener las mejores relaciones posibles con las personas que nos rodean. Una alta IE nos ayuda a ser capaces de ofrecer a los que nos rodean una información adecuada acerca de nuestro estado psicológico. Para poder manejar los estados emocionales de los demás hay que ser capaz, previamente, de manejar bien los propios estados emocionales. Las personas emocionalmente inteligentes no 
sólo son más hábiles a la hora de percibir, comprender y manejar sus propias emociones, sino también son más capaces de extrapolar estas habilidades a las emociones de los demás. En este sentido, la IE va a jugar un papel elemental en el establecimiento, mantenimiento y calidad de las relaciones interpersonales. Algunos estudios realizados han encontrado datos empíricos que apoyan la relación entre la IE y unas adecuadas relaciones interpersonales (Brackett et al., 2006; Extremera y Fernández-Berrocal, 2004; Lopes, Salovey, Cote, Beers, 2005).

\section{Inteligencia emocional y bienestar psicológico}

En la última década ha habido un conjunto de estudios que se han centrado en analizar el papel de la IE en el bienestar psicológico de los alumnos. El modelo de Mayer y Salovey nos proporciona un marco adecuado para conocer los procesos emocionales básicos que subyacen al desarrollo de un adecuado equilibrio psicológico y ayuda a comprender mejor el papel mediador de ciertas variables emocionales de los alumnos y su influencia sobre el ajuste psicológico y el bienestar personal. Los estudios realizados en Estados Unidos muestran que los alumnos universitarios con más IE informan de un menor número de síntomas físicos, menos ansiedad social y depresión, mayor utilización de estrategias de afrontamiento activo para solucionar problemas y menos rumiación. Además, cuando estos alumnos son expuestos a tareas estresantes de laboratorio, perciben los estresores como menos amenazantes y sus niveles de cortisol y de presión sanguínea son más bajos (Salovey, Stroud, Woolery y Epel, 2002) e, incluso, se recuperan mejor de los estados de ánimo inducidos experimentalmente (Salovey, Mayer, Goldman, Turvey y Palfai, 1995). Las investigaciones realizadas con adolescentes españoles muestran que cuando se les divide en grupo en función de sus niveles de sintomatología depresiva, los estudiantes con un estado normal se diferenciaban de los clasificados como depresivos por una mayor claridad hacia sus sentimientos y por una mayor capacidad para regular sus emociones (Fernández-Berrocal, Alcaide, Extremera y Pizarro, 2006; para una revisión más extensa ver Fernández-Berrocal y Extremera, 2007).

\section{Inteligencia emocional y rendimiento académico}

La capacidad para atender a nuestras emociones, experimentar con claridad los sentimientos y poder reparar los estados de ánimo negativos va a influir decisivamente sobre la salud mental de los estudiantes y este equilibrio psicológico, a su vez, está relacionado y afecta al rendimiento académico final. Las personas con escasas habilidades emocionales es más probable que experimenten estrés y dificultades emocionales durante sus estudios y, en consecuencia se beneficiarán más del uso de habilidades emocionales adaptativas que les permi- 
ten afrontar tales dificultades. La IE podría actuar como un moderador de los efectos de las habilidades cognitivas sobre el rendimiento académico (Fernández-Berrocal, Extremera y Ramos, 2003; Gil-Olarte, Palomera y Brackett, 2006; Pérez y Castejón, 2007; Petrides, Frederickson y Furnham, 2004).

\section{Inteligencia emocional y la aparición de conductas disruptivas}

Las habilidades que incluye la IE son un factor clave en la aparición de conductas disruptivas en las que subyace un déficit emocional. Es lógico esperar que los alumnos con bajos niveles de IE presenten mayores niveles de impulsividad y peores habilidades interpersonales y sociales, lo que cual favorece el desarrollo de diversos comportamientos antisociales (Extremera y Fernández-Berrocal, 2002; 2004; Mestre, Guil, Lopes, Salovey y Gil-Olarte, 2006; Petrides et al., 2004). Algunos investigadores sugieren que las personas con una menor inteligencia emocional se implican más en conductas autodestructivas tales como el consumo de tabaco (Brackett y Mayer, 2003; Brackett, Mayer y Warner, 2004; Canto, FernándezBerrocal, Guerrero y Extremera, 2005; Trinidad y Johnson, 2002; Trinidad, Unger, Chou y Johnson, 2004a; Trinidad, Unger, Chou y Johnson, 2004b; Trinidad, Unger, Chou y Johnson, 2005). Los adolescentes con una mayor capacidad para manejar sus emociones son más capaces de afrontarlas en su vida cotidiana facilitando un mejor ajuste psicológico y teniendo así un menor riesgo de consumo abusivo de sustancias. En concreto, los adolescentes con un mayor repertorio de competencias afectivas basadas en la comprensión, el manejo y la regulación de sus propias emociones no necesitan utilizar otro tipo de reguladores externos (e.g., tabaco, alcohol y drogas ilegales) para reparar los estados de ánimo negativos provocados por la variedad de eventos vitales y acontecimientos estresantes a los que se exponen en esas edades (Ruiz-Aranda, Fernández-Berrocal, Cabello y Extremera, 2006).

\section{¿Es posible educar la IE?}

Tras esta exposición resumida de los beneficios de la IE en diferentes dominios del contexto escolar el lector probablemente pensará: "Es muy útil tener estas habilidades en la escuela. De acuerdo, y el mundo sería perfecto si mis alumnos poseyeran estas habilidades emocionales y sociales, pero ¿y si no es así?” Y la siguiente pregunta que surge, de forma inevitable, es: ¿Puedo educar la IE de mis alumnos? 
Educar la inteligencia emocional se ha convertido en una tarea necesaria en el ámbito educativo y la mayoría de los padres y docentes considera primordial el dominio de estas habilidades para el desarrollo evolutivo y socioemocional de sus hijos y alumnos. No obstante, hay muchas formas de llevarlo a cabo y, desde nuestro punto de vista, es muy importante enseñar a los niños y a los adolescentes programas de IE que de forma explícita contengan y resalten las habilidades emocionales basadas en la capacidad para percibir, comprender y regular las emociones, como destaca el modelo de Mayer y Salovey (Grewal y Salovey, 2005; Mayer y Salovey, 1997).

La enseñanza de estas habilidades depende de forma prioritaria de la práctica, el entrenamiento y su perfeccionamiento, y no tanto de la instrucción verbal. Lo esencial es ejercitar y practicar las habilidades emocionales y convertirlas en una respuesta adaptativa más del repertorio natural de la persona.

¿Son eficaces este tipo de ejercicios en IE? Sí, aunque no son muchos, existen estudios contrastados que apoyan la eficacia de programa específicos de entrenamiento en las habilidades emocionales que componen la IE (Lopes y Salovey, 2004; Maurer y Brackett, 2004; ver también, Fernández-Berrocal y Ramos, 2004). En concreto, en el ámbito educativo los programas llevados a cabo en EE.UU. bajo la denominación de SEL han mostrado unos resultados muy prometedores (Weissberg y O’Brien, 2004; ver también, en este monográfico RuizAranda, Fernández-Berrocal, Cabello y Salguero, 2008).

\section{Conclusión}

El pequeño Alfred Binet habría agradecido que su profesora Martha hubiera tenido la IE suficiente para señalarle su error, pero sin esa carga de emociones destructivas con las que de forma imprudente lo hizo. La sociedad europea en la que nació y vivió Binet ha cambiado de forma vertiginosa y ya casi no es reconocible desde nuestra mentalidad de siglo XXI. En cambio, la escuela en la que se formó Binet, aún pervive en alguno de sus más aspectos menos evolucionados en nuestro sistema educativo actual.

Paises tan representativos del G8 (el "club de los países más ricos") como Estados Unidos y el Reino Unido han comenzado a comprender que ser uno de los ocho países del mundo más industrializados, ricos e influyentes del planeta no garantiza que sus ciudadanos 
estén satisfechos con sus vidas y sean felices. En España la situación no es aún tan crítica como en estos países pero, más allá de las estadísticas y los barómetros de bienestar, cualquier observador atento de la realidad cotidiana de la escuela y de nuestra sociedad puede observar que nos acercamos peligrosamente a sus niveles de insatisfacción e infelicidad.

Estados Unidos y el Reino Unido están reaccionando de forma apropiada a este desafío tanto desde instituciones públicas como privadas. Para ello, están apoyando con cuantiosos fondos de investigación el estudio, la aplicación y evaluación de diferentes programas de educación socioemocional en la escuela. En este sentido, España está en un momento excelente para hacer políticas e inversiones educativas similares a las emprendidas por estos países. No obstante, no debieramos hacerlas de forma precipitada y descoordinada como el ritmo de la urgencia suele imponer en nuestro país, sino desde una perspectiva sería y rigurosa, que nos permita hacer un desarrollo extenso de la educación socioemocional pensando a medio y a largo plazo.

Quizá las contradicciones y paradojas de las sociedades hipermodernas no puedan ser resueltas sólo mediante la educación de las emociones en las escuelas y sean necesarios otros cambios radicales en nuestro entorno y en nuestras vidas para resolverlos, pero no cabe duda de que el siglo XXI será un tiempo más amable y estimulante para todos con futuros ciudadanos con más IE.

\section{Referencias}

Binet, A. (1909). Las ideas modernas acerca de los niños. Alicante: Biblioteca Virtual Miguel de Cervantes (trad. cast., 1999).

Brackett, M. A., y Mayer, J.D. (2003). Convergent, discriminant and incremental validity of competing measures of emotional intelligence. Personality and Social Psychology Bulletin, 29, 1147-1158.

Brackett, M., Mayer, J.D. y Warner, R.M. (2004). Emotional intelligence and the prediction of behavior. Personality and Individual Differences, 36, 1387-1402.

Brackett, M., Rivers, SE., Shiffman, S., Lerner N. y Salovey, P. (2006). Relating emotional abilities to social functioning: a comparison of self-report and performance measures of emotional intelligence. Journal of Personality and Social Psychology, 91,780-795.

Canto, J., Fernández-Berrocal, P., Guerrero, F., y Extremera, N. (2005). Función protectora de las habilidades emocionales en las adicciones. En J. Romay Martínez y R. García Mira (Eds), Psicología Social y Problemas Sociales (pp. 583-590). Madrid: Biblioteca Nueva. 
Ciarrochi, J.V., Chan, A. y Bajgar, J., (2001). Measuring emotional intelligence in adolescents. Personality and Individual Differences, 31, 1105-1119.

Extremera, N. y Fernández-Berrocal, P. (2002). La inteligencia emocional en el aula como factor protector de conductas problema: violencia, impulsividad y desajuste emocional. En F. A. Muñoz, B. Molina y F. Jiménez (Eds.) Actas I Congreso Hispanoamericano de Educación y Cultura de Paz (pp. 599-605). Editorial Universidad de Granada: Granada.

Extremera, N. y Fernández-Berrocal, P. (2003). La inteligencia emocional en el contexto educativo: Hallazgos científicos de sus efectos en el aula. Revista de Educación, 332, 97116.

Extremera, N. y Fernández-Berrocal, P. (2004). El papel de la inteligencia emocional en alumnado: evidencias empíricas. Electronic Journal of Resarch of Educational Psychology, 6 (2), 363-382.

Evans, D. (2002). Emoción. La ciencia del sentimiento. Madrid: Taurus.

Fernández, M.R., Palomero, J.E., Teruel, M.P. (2008). El desarrollo socioafectivo en la formación inicial de los maestros. Electronic Journal of Research in Educational Psychology, 14.

Fernández-Berrocal, P., Alcaide, R., Extremera, N. y Pizarro, D. A. (2006). The role of emotional intelligence in anxiety and depression among adolescents. Individual Differences Research, 4, 16-27.

Fernández-Berrocal, P. y Extremera, N. (2007). Inteligencia emocional y salud. En Mestre, J.M. y Fernández-Berrocal, P. (Eds), Manual de Inteligencia emocional, (pp. 173187). Madrid: Ed. Pirámide.

Fernández-Berrocal, P. y Ramos, N. (2002). Corazones Inteligentes. Barcelona: Kairós.

Fernández-Berrocal, P. y Ramos, N. (2004). Desarrolla tu Inteligencia Emocional. Barcelona: Kairós.

Goleman, D. (1995). Inteligencia Emocional. Barcelona: Kairós.

Greenberg, M. T., Weissberg, R. P., O’Brien, M. U., Zins, J. E., Fredericks, L., Resnik, H., y Elias, M. J. (2003). Enhancing school-based prevention and youth development through coordinated social, emotional, and academic learning. American Psychologist, $58,466-74$.

Grewal, D., y Salovey, P. (2005). Feeling Smart: The Science of Emotional Intelligence. American Scientist, 93, 330-339.

Gil-Olarte, P., Palomera, R. y Brackett, M. (2006). Relating emotional intelligence to social competence and academic achievement in high school students. Psicothema, 18, supl., 118-123.

Instituto Nacional de Estadística (2007). INEbase. Obtenido en http://www.ine.es/inebmenu/indice.htm 
Lipovetsky, G. (2006). Los tiempos hipermodernos. Barcelona: Ed. Anagrama.

Lopes, P., y Salovey, P. (2004). Toward a broader education: Social, emotional, and practical skills. En J.E. Zins, R.P. Weissberg, M.C. Wang, y H.J. Walberg (Eds.), Building school success on social and emotional learning (pp. 79-93). New York: Teachers College Press.

Lopes, L., Salovey, P., Cote, S. y Beers, M., (2005). Emotion regulation abilities and the quality of social interaction. Emotion, 5, 113-118.

Maurer, M. y Brackett M. A., (2004). Emotional Literacy in the middle school. A 6-step program to promote social, emotional and academic learning. New York: Ed. Dude.

Mayer, J.D. y Salovey, P. (1997). What is emotional intelligence? En P. Salovey y D. Sluyter (eds.). Emotional Development and Emotional Intelligence: implications for educators (pp. 3-31). New York: Basic Books.

Mayer, J.D., Salovey, P. y Caruso, D. (2000). Models of emotional intelligence. En R.J. Sternberg (ed.), Handbook of intelligence (pp. 396-420). New York: Cambridge.

Mestre, J.M., y Fernández-Berrocal, P. (2007). Manual de Inteligencia Emocional. Madrid: Pirámide.

Mestre, J.M., Guil, R., Lopes, P., Salovey, P. y Gil-Olarte, P. (2006). Emotional Intelligence and social and academia adaptation to school. Psicothema, 18, 112-117.

Ministerio Sanidad y Consumo (2007). Informe de la encuesta estatal sobre uso de drogas en estudiantes de enseñanzas secundarias (Estudes) 2006-2007. Madrid: MSC.

Palomera, R., Fernández-Berrocal, P. y Brackett, M. (2008). La inteligencia emocional como una competencia básica en la formación inicial de los docentes: algunas evidencias. Electronic Journal of Research in Educational Psychology, 14.

Pérez, N. y Castejón, J.L. (2007). La inteligencia emocional como predictor del rendimiento académico en estudiantes universitarios. Ansiedad y estrés, 13 (1), 119-129.

Petrides, K. V., Frederickson, N. y Furnham, A. (2004). The role of trait emotiona intelligence in academic performance and deviant behaviour at school. Personality and Individual Differences, 36 (2), 277-293.

Ruiz-Aranda, D., Fernández-Berrocal, P., Cabello, R. y Extremera, N. (2006). Inteligencia emocional percibida y consumo de tabaco y alcohol en adolescentes. Ansiedad y estrés, 12 (2-3), 223-230.

Ruiz-Aranda, D., Fernández-Berrocal, P., Cabello, R. y Salguero, N. (2008). Educando la inteligencia emocional en el aula: Proyecto Intemo. Electronic Journal of Research in Educational Psychology, 14.

Salovey, P. y Mayer, J. D. (1990). Emotional intelligence. Imagination, Cognition, and Personality, 9, 185-211. 
Salovey, P., Mayer, J.D., Goldman, S.L., Turvey, C. y Palfai, T.P. (1995). Emotional attention, clarity, and repair: exploring emotional intelligence using the Trait Meta-Mood Scale. En J.W. Pennebaker (ed.), Emotion, Disclosure y Health (pp.125-151). Washington: American Psychological Association.

Salovey, P., Stroud, L.R., Woolery, A. y Epel, E.P. (2002). Perceived emotional intelligence, stress reactivity, and symptoms reports: further explorations using the Trait MetaMood Scale. Psychology and Health, 17, 611-627.

Sánchez Núñez, M. T.; Fernández-Berrocal, P.; Montañés, J. \& Latorre, J. M. (2008). Does emotional intelligence depend on gender? The socialization of emotional competencies in men and women and its implications. Electronic Journal of Research in Educational Psychology, 14.

Trinidad, D.R. y Johnson, C.A. (2002). The association between emocional intelligence and early adolescent tobacco and alcohol use. Personality \& Individual Differences, 32 95105.

Trinidad, D. R., Unger, J.B., Chou, C.P., y Johnson, C.A. (2004a). The protective association between emotional intelligence with psychosocial smoking risk factors for adolescents. Personality and Individual Differences, 36, 945-954.

Trinidad, D. R., Unger, J.B., Chou, C.P., y Johnson, C.A. (2004b). Emotional Intelligence and Smoking Risk Factors in Adolescents: Interactions on Smoking Intentions. Journal of Adolescent Health, 34, 46-55.

Trinidad, D. R., Unger, J.B., Chou, C.P., y Johnson, C.A. (2005). Emotional Intelligence and Acculturation to the United States: Interactions on the Perceived Social Consequences of Smoking in Early Adolescents. Substance Use and Misuse, 40, 1697-1706.

UNICEF (2007). Pobreza infantil en perspectiva: Un panorama del bienestar infantil en los países ricos, Innocenti Report Card. Florencia: Centro de Investigaciones Innocenti de UNICEF.

Weissberg, R. P., y O'Brien, M. U. (2004). What works in school-based social and emotional learning programs for positive youth development. The Annals of the American Academy of Political and Social Science, 591, 86-97.

Zins, J.E., Weissberg, R.P., Wang, M.C., y Walberg, H.J. (Eds.). (2004). Building academic success on social and emotional learning: What does the research say? New York: Teachers College Press. 